\title{
Accurate Measurement of Energy Dissipated in Braking Rheostats in DC Railway Systems
}

\author{
Helko van den Brom ${ }^{1}$, Domenico Giordano ${ }^{2}$, Danielle Gallo ${ }^{3}$, Andreas Wank ${ }^{4}$, Yljon Seferi ${ }^{5}$ \\ ${ }^{1}$ VSL, ${ }^{2}$ INRIM, ${ }^{3}$ University of Campania, ${ }^{4}$ NPL, ${ }^{5}$ University of Strathclyde \\ hvdbrom@vsl.nl
}

\begin{abstract}
This paper describes the measurement system used to monitor the energy dissipated in the braking rheostat resistors on board a locomotive operating in a DC rail system. The aim of the activity is the accurate estimation of the energy, nowadays wasted, that could be recovered thanks to the implementation of the smart grid paradigm in the DC railway system. To provide metrological reliability to the measured wasted energy, a preliminary identification and estimation of the uncertainty is provided. The target uncertainty of the measurements is $1 \%$.
\end{abstract}

Index Terms - Current measurement, energy measurement, rail transportation, transducers, measurement uncertainty.

\section{INTRODUCTION}

The estimation of the energy dissipated in on-board braking rheostats (BR) during the braking stage of a train has a significant impact on the investment decisions for a sustainable transport system. This is especially relevant for DC railway systems which usually cannot deliver the energy to the upstream supply system. This energy is nowadays indirectly estimated starting from the knowledge of the mechanical braking energy and the energy sent back to the catenary and absorbed by other trains. This procedure, however, does not provide very reliable figures. Accurate measurements are necessary to provide real evidence on the actual amount of energy consumption and $\mathrm{CO}_{2}$ emissions that can be reduced.

Direct and accurate measurement of the energy dissipated in the BR is challenging from the metrological point of view. The rheostats experience a chopped current that switches with a frequency of hundreds of hertz from zero to, in the most extreme cases, thousands of amperes in some microseconds. To estimate the amount of energy lost, the voltage and current signals need to be accurately measured at the BR during chopping. In this paper, we demonstrate such measurements for a DC traction unit, determine the actual amount of energy wasted and calculate the related measurement uncertainty. The target uncertainty of these measurements is $1 \%$.

\section{ON-BOARD MEASUREMENT SYSTEM}

A measurement campaign has been performed on the E464-041 from Trenitalia, a 3.5 MW locomotive operating in a $3 \mathrm{kV}$ DC power supply system in the north-west of Italy. Five electrical quantities have been recorded for several months at a sampling frequency of $50 \mathrm{kHz}$ using a data acquisition system installed on-board the train. One of the aims of the campaign was the accurate estimation of the energy wasted at the BR.
The operating mode of the BR is dependent on the voltage level of the pantograph: for voltages below $3800 \mathrm{~V}$, the braking energy is sent back to the catenary and used elsewhere, above $3900 \mathrm{~V}$ the electrical braking energy is dissipated in the BR, and between $3800 \mathrm{~V}$ and $3900 \mathrm{~V}$ the energy is a mixture of both. The amount of energy dissipation is controlled by a chopper circuit varying the duty-cycle. The frequency of the chopping is fixed at $195 \mathrm{~Hz}$ whereas the duty-cycle can vary from $0 \%$ to $50 \%$. Two BR resistors are supplied by two traction inverters, connected in series as presented in Figure 1. To guarantee that each motor experiences the same braking effort, the two BR resistors are alternatively connected to the two inverters with each pulse.

The measurement set-up is described in [1]. The actual chopped current $I_{a-c h o p}$ and $I_{b-c h o p}$ is measured directly at the BR resistors $R_{a}$ and $R_{b}$. The voltage signals are the continuous pantograph voltage measured after the downstream-filter, $V_{d s f}$, and the half-filter voltage $V_{h f}$ measured between the rheostats.

\section{DETERMINING THE DISSIPATED ENERGY}

The easiest way to determine the energy dissipated in the BR is to take the time integral of the power calculated from the measured instantaneous current through both BR resistors and their respective resistance values. However, the uncertainty of the resistance is of the order of $5 \%$, partly due to the local temperature that can increase to a few hundred degrees Celsius when braking.

A more accurate way to determine the dissipated energy is by calculating the power using the instantaneous current through the BR resistors and the measured voltage. Figure 2 shows the time behavior of the voltage and current signals during the initial stage of the chopping process for resistor $R_{a}$, when the

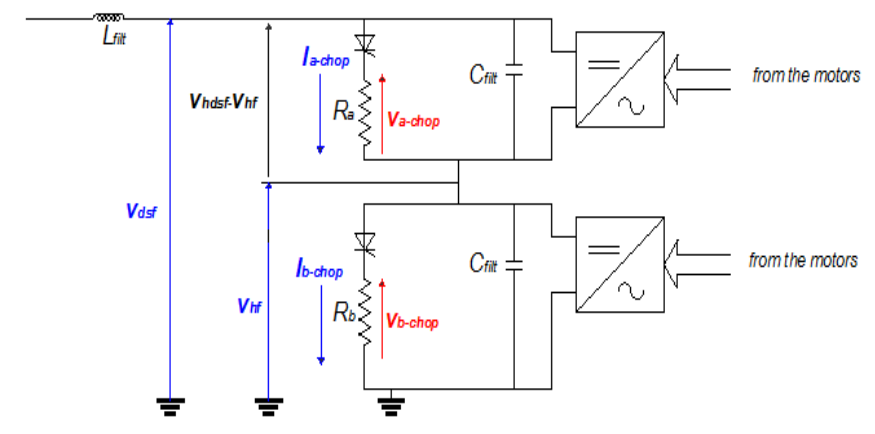

Fig. 1. Simplified electrical diagram of the motor inverters and braking rheostats of the E464 locomotive. 
duty cycle is low. The sequence of alternating the resistor connections is mathematically implemented by alternately multiplying successive current pulses by the voltage signals $\left(V_{d s f}-V_{h f}\right)$ and $V_{h f}$, respectively.

To measure the voltage and current signals, voltage transducers are used with voltage ratio $k$ and current transducers with transresistance ratio $R_{\text {trans }}$, the latter resulting in ADC readings $V_{\text {trans }}^{a}$ and $V_{\text {trans }}^{b}$. The dissipated energy is calculated using a discrete form of the earlier mentioned integral equation:

$$
E=\sum_{i=0}^{N}\left(\left(V_{d s f, i}-V_{h f, i}\right) \cdot \frac{V_{\text {trans }, i}^{a}}{R_{\text {trans }}^{a}}+V_{h f, i} \cdot \frac{V_{\text {trans }, i}^{b}}{R_{\text {trans }}^{b}}\right) \cdot \Delta t .
$$

\section{UNCERTAINTY OF THE DISSIPATED ENERGY}

Each of the quantities indicated in the above-mentioned equation has its own uncertainty due to calibration of transducers [2] or ADCs. Furthermore, the ADC readings, corresponding to the relevant voltage and current signals, respectively, have their own Type A contribution. Apart from these obvious terms, a few other terms come into play.

The current through the rheostats is measured at the position where the energy is dissipated, whereas the voltage is the downstream-filter voltage and the half-filter voltage, respectively, i.e., before the choppers. The difference between the measured voltage and the actual voltage over the rheostat resistors $V_{a-c h o p}$ and $V_{b-c h o p}$ is denoted $\delta V_{d s f, p o s}$ and $\delta V_{h f \text {,pos, }}$, respectively, and is taken into account as a separate uncertainty contribution. Since this difference is mainly due to voltage drop over the cable resistance, its effect is negligible considering the resistance of the rheostat which is on the order of an ohm.

Another lacking piece of information is the starting configuration of the choppers. If, at the first switching pulse, resistor $R_{a}$ is connected to the upper inverter (as in Figure 1), the voltage that must be used for power estimation is $\left(V_{d s f}-V_{h f}\right)$, whereas for the second pulse, the voltage is $V_{h f}$. Since we do not know which of the two voltages to multiply, we calculate the power $P_{a b}$ and $P_{b a}$ for both cases, and calculate the difference:

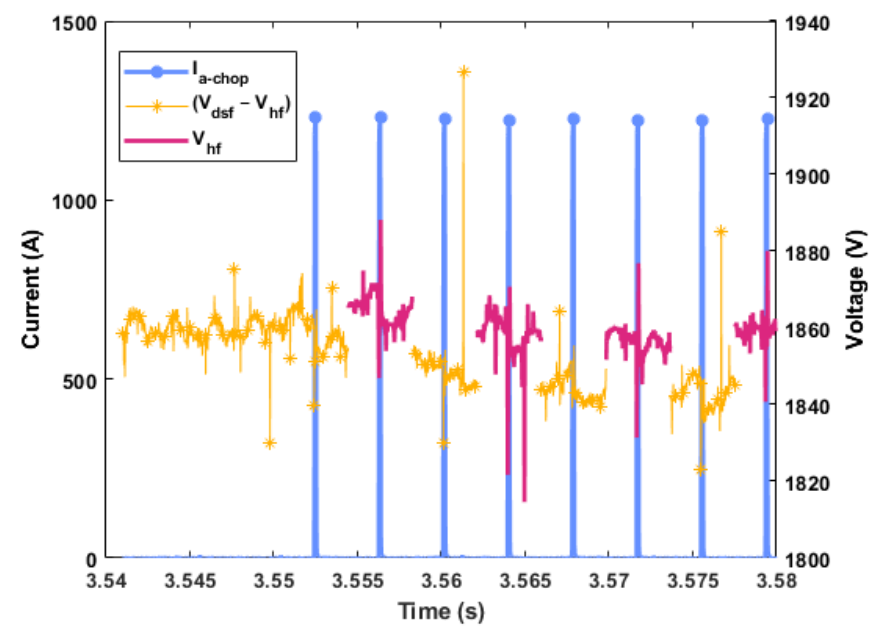

Fig. 2. Measured chopped current and voltage signals modified for the alternate multiplication with the current as explained in the text.

$$
\Delta P_{a b} \equiv P_{a b}-P_{a b}=\left(I_{a}-I_{b}\right) \cdot\left(V_{d s f}-2 \cdot V_{h f}\right) .
$$

Hence, the difference between the two situations is negligible if the two currents are equal or if twice the half-filter voltage is equal to the downstream-filter voltage, as expected. In case there is a difference of, say, a few percent between the two currents and a difference of a few percent between the two voltages, the effect on the difference in power $\Delta P_{a b}$ is only about $0.1 \%$. Note that the uncertainty due to this difference, $\delta \Delta P_{a b}$, does not show a normal probability distribution.

Apart from the BR resistors, energy is also dissipated in other components on-board the train, such as the choppers themselves. The latter is captured in an extra uncertainty contribution $\delta \Delta P_{\text {other }}$.

Most of the terms described above can be determined with an estimated uncertainty contribution on the order of $0.1 \%$ when calibrated carefully, resulting in an overall uncertainty of about $1.0 \%$. However, these results are strongly dependent on the equipment used. For instance, non-calibrated transducers have typical uncertainties of about $2 \%$, and the temperature dependence of the current or voltage transducers might be significantly worse than $0.1 \%$ over the relevant temperature range. Furthermore, the effect of the position of the voltage measurement might be worse, which would lead to the conclusion that the voltage should also be measured at the rheostat itself.

\section{CONCLUSION}

Accurate measurements were performed to determine the energy dissipated in braking rheostat resistors on-board a DC railway traction unit. An uncertainty estimation was presented describing a wide range of uncertainty contributions. Future work will concentrate on determining the uncertainty contributions in more detail. Furthermore, software will be developed to calculate the exact uncertainty. The target uncertainty of $1.0 \%$ seems to be within reach.

\section{ACKNOWLEDGEMENT}

The research leading to the results described in this paper is performed within the 16ENG04 MyRails project of the European Metrology Programme for Innovation and Research (EMPIR). The EMPIR initiative is co-funded by the European Union's Horizon 2020 research and innovation programme and the EMPIR participating states.

\section{REFERENCES}

[1] G. Crotti et al., "Monitoring Energy and Power Quality On Board Train", IEEE International Workshop on Applied Measurements for Power Systems (AMPS), Aachen, Germany, 2019, pp. 1-6.

[2] H. E. van den Brom, R. van Leeuwen, and R. Hornecker, "Characterization of DC current sensors with AC distortion for railway applications", IEEE Trans. Instrum. Meas., Vol. 68, pp. 2084-2090, June 2019. 